\section{Rapid detection of Mycobacterium tuberculosis complex in cattle and lechwe (Kobus leche kafuensis) at the slaughter house}

\author{
Mudenda B. Hang'ombe, 1 \\ Chie Nakajima,2 Akihiro Ishii,2 \\ Yukari Fukushima,2 Musso Munyeme, 1 \\ Wigganson Matandiko, ${ }^{3}$ \\ Aaron S. Mweene, ${ }^{1}$ Yasuhiko Suzuki2,4 \\ 1School of Veterinary Medicine, \\ University of Zambia, Lusaka, Zambia; \\ 2Hokkaido University Research Center for \\ Zoonosis Control, Sapporo, Hokkaido, \\ Japan; \\ 3Zambia Wildlife Authority, Private Bag \\ 001, Chilanga, Zambia; \\ 4JST/JICA-SATREPS
}

\section{Abstract}

The detection and diagnosis of tuberculosis (TB) in food-producing animals is critical to human health. In this study we applied the loop-mediated isothermal amplification (LAMP) system to detect Mycobacterium tuberculosis complex (MTC) directly in 57 cattle and six lechwe (Kobus leche kafuensis) carcasses exhibiting lesions characteristic of TB. The samples were first subjected to Ziehl-Neelsen microscopy, followed by culture and LAMP assay. In addition, multiplex-PCR was used to determine the species involved. Of the samples from the cattle, $84.2 \%$ (95\% confidence interval: 71.6-92.1) were found positive with ZiehlNeelsen microscopy, 93.0\% (95\% confidence interval: 82.2-97.7) with culture, and $94.7 \%$ (95\% confidence interval: 84.5-98.6) with the LAMP system while the Kobus leche kafuensis samples were all positive for all techniques used. These results indicate that the LAMP system can be used to augment the detection and surveillance of TB in animals; hence can be a very useful tool in the veterinary field and in public health.

\section{Introduction}

The detection and diagnosis of tuberculosis (TB) in animals involves clinical examination, tuberculin skin tests, and the gamma interferon immunoassay.1,2 Other techniques involve detection on meat inspection or postmortem followed by the usual course of microscopic observation of acid-fast bacilli, bacterial cultures, and molecular confirmation methods. ${ }^{3}$
TB in animals can be caused by Mycobacterial species belonging to the Mycobacterium tuberculosis complex (MTC). This group comprises several closely related species responsible for strictly human and zoonotic TB. These include M. tuberculosis, M. africanum, M. microti, $M$. bovis, $M$. caprae, and $M$. pinnipedii. ${ }^{4-6}$ Some of these Mycobacterium species are major reemerging zoonotic agents of bovine TB, the prevalence of which depends on direct exposure to cattle and consumption of unpasteurized dairy products. ${ }^{7,8}$ Furthermore, the interaction in the human-livestock-wildlife interphase areas of some wildlife animals like lechwe (Kobus leche kafuensis), documented with $M$. bovis, have broadened the reservoir base for MTC. 9

In this regard, detection and diagnosis of TB is very important as a way of mitigating its spread in the human population. Currently, a novel molecular amplification method termed the loop-mediated isothermal amplification system (LAMP) has been developed. 10 The system shows a high amplification efficiency and has been used to diagnose several other diseases. ${ }^{10-17}$ The objective of our study was to evaluate the applicability of the LAMP system, in the veterinary field, to detect MTC directly from suspected TB lesions of cattle and wildlife being slaughtered for food.

\section{Materials and Methods}

\section{Sampling}

Our study was conducted on samples collected from the slaughtered animals along the examination line. The animals were examined for gross lesions according to the standard postmortem procedures as described previously. 18 Organs and tissues with suspected TB lesions were collected after detailed postmortem examination of the entire carcasses. Following collection, the specimens were placed into a cooler box with ice packs before transportation to the laboratory for analysis.

\section{Preparation of samples for evaluation}

To prepare the suspected TB samples for analysis, the suspected tissues with lesions were trimmed of fat and then a 500-mg sample was collected. The sample was then minced with sterile scissors and homogenized in a sterilized glass homogenizer, after which $1 \mathrm{~mL}$ of phosphate buffer ( $\mathrm{pH} 6.8$ ) was added. After thorough mixing, $1 \mathrm{~mL}$ of $5 \%$ sodium hydroxide was added. This was mixed thoroughly and then incubated for 15 min at room temperature. To this mixture $10 \mathrm{~mL}$ of phosphate buffer was added and then centrifuged at 1500 $g$ for $20 \mathrm{~min}$. The pellet was collected and then resuspended in a final volume of $0.5 \mathrm{~mL}$ of
Correspondence: Mudenda Bernard Hang'ombe, School of Veterinary Medicine, Department of Paraclinical Studies, P. 0. Box 32379, Lusaka, Zambia.

E-mail: mudenda68@yahoo.com

Key words: Mycobacterium bovis, cattle, Kobus leche kafuensis, LAMP, multiplex-PCR.

Acknowledgments: the authors are grateful to $\mathrm{Mr}$ L. Moonga and $\mathrm{Mr}$ E. Mulenga from the Department of Paraclinical Studies, School of Veterinary Medicine, University of Zambia for their technical assistance. This work was supported by the Directorate of Research and Graduate Studies of the University of Zambia, the Ministry of Education, Culture, Sports, Science and Technology of Japan (MEXT), and the Global Center of Excellence (COE) program for the Establishment of International Collaboration Centers for Zoonoses control, by grants from the US-Japan Cooperative Medical Science program to YS and the Japan Society for the Promotion of Science to YS and $\mathrm{CH}$.

Contributions: $\mathrm{MBH}$, sample collection, isolation, LAMP and PCR detection, analysis, data interpretation, and manuscript drafting; CN, AI, YF, MM, WM, design of study, sample collection, and manuscript drafting; ASM, YS, interpretation of data, drafting, and final approval of manuscript.

Conflict of interest: the authors report no conflicts of interest.

Received for publication: 3 March 2011. Accepted for publication: 29 April 2011.

This work is licensed under a Creative Commons Attribution 3.0 License (by-nc 3.0).

(C) Copyright M.B. Hang'ombe et al., 2010 Licensee PAGEPress, Italy

Veterinary Science Development 2011; 1:e5 doi:10.4081/vsd.2011.e5

phosphate buffer. This was used for inoculation into $2 \%$ Ogawa medium and preparation of slides for Ziehl-Neelsen (ZN) microscopy. Cultures were monitored for growth up to $8 \mathrm{wk}$ at $37^{\circ} \mathrm{C}$. Another $100 \mu \mathrm{L}$ of the suspension was used to prepare DNA directly by using DNAzol reagent (Invitrogen, Carlsbad, CA, USA). The suspension was mixed with $1.0 \mathrm{~mL}$ of DNAzol reagent and mechanical disruption was used as previously described. ${ }^{19}$ DNA was extracted according to the manufacturer's instructions. Genomic DNA from Mycobacterium bacterial cultures was also prepared from colonies using DNAzol and mechanical disruption as described earlier. The extracted DNA was then dissolved in $50 \mu \mathrm{L}$ TE buffer consisting of 10 mM Tris/HCL (pH 8.0) and 1 mM EDTA. 


\section{LAMP}

LAMP reactions were performed in a total volume of $25 \mu \mathrm{L}$ consisting of $30 \mathrm{pmol}$ each of inner primers FIP and BIP, 5 pmol each of outer primers F3 and B3, 20 pmol each of loop primers FLP and BLP, 1.4 mM deoxynucleotide triphosphate, $0.8 \mathrm{M}$ betaine, $20 \mathrm{mM}$ Tris/ $\mathrm{HCl}$ (pH 8.8), $10 \mathrm{mM} \mathrm{KCl}, 10 \mathrm{Mm}\left(\mathrm{NH}_{4}\right)_{2} \mathrm{SO}_{4}, 8 \mathrm{mM}$ $\mathrm{MgSO}_{4}$ and $8 \mathrm{U}$ Bst DNA polymerase (New England Biolabs, USA) with $2 \mu \mathrm{L}$ sample DNA. The sequences of the primers used are shown in Table 1.

The primers used in our study have also been used by other workers to detect MTC in human sputum samples. 17 The mixture was then incubated at $64^{\circ} \mathrm{C}$ for $1 \mathrm{hr}$ in a thermal dry heat block (ALB 221; Iwaki, Tokyo, Japan). A negative control (buffer) and positive control were included in each run. Results were visualized with the fluorescence detection reagent (Eiken Chemical Co., Tochigi, Japan) according to the manufacturer's instructions.

\section{Multiplex-PCR primers and condi- tions}

Genomic DNA from Mycobacterium bacterial cultures was used as a template. Primer pairs for $c f p 32$ (a specific gene for MTC), RD9 (region of difference 9 seen only in $M$. tuberculosis and M. canettii), and RD12 (region of difference 12 deleted in $M$. bovis, $M$. caprae, and $M$. canettii) were obtained from an earlier publication. ${ }^{20}$ The general PCR recipe contained $7.4 \mu \mathrm{L} \mathrm{H} \mathrm{H}_{2} \mathrm{O}, 2 \mu \mathrm{L} 10 \times \mathrm{Taq}$ buffer, $2 \mu \mathrm{L}$ dNTPs (2.5 mM each), $0.2 \mu \mathrm{L}$ Taq (Takara), $1 \mu \mathrm{L}$ target DNA, $2.2 \mu \mathrm{L}$ of $10 \mu \mathrm{M}$ cfp32 primers, $0.7 \mu \mathrm{L}$ of $5 \mu \mathrm{M}$ RD9 primers, and $0.8 \mu \mathrm{L}$ of $5 \mu \mathrm{M}$ RD12 primers. Appropriate negative controls consisting of PCR mix without target DNA were included. The PCR was performed using the following program: denaturation for $1 \mathrm{~min}$ at $98^{\circ} \mathrm{C}$ followed by 35 cycles of $5 \mathrm{sec}$ at $98^{\circ} \mathrm{C}, 20$ sec at $58^{\circ} \mathrm{C}$, and $1 \mathrm{~min}$ at $68^{\circ} \mathrm{C}$ with final extension for $5 \mathrm{~min}$ at $72^{\circ} \mathrm{C}$ in a thermalcycler (iCycler, Bio-Rad Laboratories Inc., CA, USA). All PCR products were identified by gel electrophoresis in a $2.0 \%$ agarose gel and were visualized by ethidium bromide staining.

\section{Results}

A total of 388 carcasses were examined, comprising 358 cattle and 30 lechwe. Of these animals examined, 57 (15.9\% CI: 12.4-20.2) cattle carcasses had lesions characteristic of TB in the tissues and organs while $6(20 \% \mathrm{CI}$ : 8.40-39.1) lechwe carcasses had such lesion exhibitions as well. When these samples were subjected to ZN microscopy, 48 (84.2\% CI: 71.692.1) and 6 (100\% CI: 51.7-100) samples from the cattle and lechwe, respectively, were found
Table 1. Primers for the specific detection of the Mycobacterium tuberculosis complex used in the study.

\begin{tabular}{ll} 
Name of primer & Sequence \\
FIP & CACCCACGTGTTACTCATGCAAGTCGAACGGAAAGGTCT \\
BIP & TCGGGATAAGCCTGGACCACAAGACATGCATCCCGT \\
\hline F3 & CTGGCTCAGGACGAACG \\
B3 & GCTCATCCCACACCGC \\
\hline FLP & GTTCGCCACTCGAGTATCTCCG \\
BLP & GAAACTGGGTCTAATACCGG \\
\hline
\end{tabular}

Table 2. Number and percentage of samples testing positive for Mycobacterium tuberculosis under different procedures.

\begin{tabular}{lccc} 
Sample source & Ziehl-Neelsen microscopy & Culture & LAMP \\
Cattle & $48(84.2 \%)$ & $53(93.0 \%)$ & $54(94.7 \%)$ \\
Lechwe & $6(100 \%)$ & $6(100 \%)$ & $6(100 \%)$ \\
\hline Total & 54 & 59 & 60 \\
Sensitivity & $85.7 \%$ & $93.7 \%$ & $95.2 \%$ \\
\hline $95 \%$ CI & $74.1-92.9$ & $83.7-97.9$ & $85.8-98.8$ \\
\hline
\end{tabular}

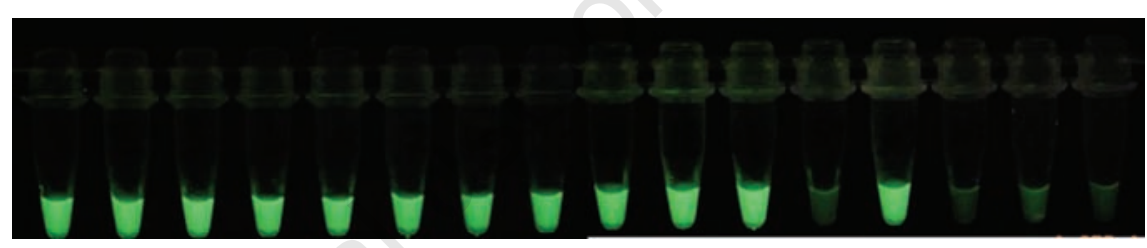

Figure 1. Visual detection of LAMP products under the UV light. From left to right, tubes12,14,15, and 16 are negative, while the rest are positive.

positive. In the case of the cattle, 9 samples were found to be negative, from those observed with characteristic TB lesions. On culture of postmortem TB-positive samples, $53(93.0 \%$ CI: 82.2-97.7) cattle and all lechwe observed at meat inspection were found to be positive for acid-fast bacilli. The positive sample specimens increased in number with the LAMP system. Furthermore, the positive reactions on LAMP were easy to determine with the naked eye, as shown in Figure 1.

The overall results for the detection of TBpositive samples are shown in Table 2 . All of the culture positive samples were positive on the LAMP assay. The culture isolates were also subjected to the LAMP system and were found to be MTC. In terms of sensitivity, the LAMP system was $100 \%$ positive in culture positive specimens. An additional sample not detected with culture was also found to be positive. The sensitivity of ZN microscopy was found to be $85.7 \%$, while culture was $93.7 \%$, and LAMP $95.2 \%$, respectively. In all these diagnostic procedures, sensitivity was taken as the proportion of samples testing positive for Mycobacterium tuberculosis.

The multiplex-PCR was successfully used to identify the MTC species involved in cattle and lechwe. The amplicon of 786bp of the $c f p 32$ region was observed as indicated in Figure 2,

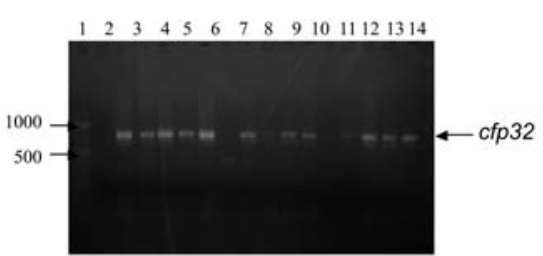

Figure 2. Multiplex-PCR products analyzed with $2 \%$ agarose gel electrophoresis followed by ethidium bromide staining. Lane 1 is a 50 bp ladder and lane 13 is a negative control.

showing that the MTC species involved in these cases was M. bovis. All of the cultures were positive for this amplicon indicating the MTC strain in the domestic and wildlife animals to be M. bovis.

\section{Discussion}

We successfully applied the LAMP assay technique for the rapid detection of the MTC group directly from clinical samples. It is important to note that the diagnosis of TB in 
animals largely depends on the tuberculin skin testing and slaughterhouse surveillance for undetected infections. ${ }^{1}$ In addition, it would be useful to develop a rapid and sensitive method to apply to doubtful TB-suspected samples. Slaughterhouse surveillance of TB is one of the key tools for detecting and confirming suspected TB cases. This is aided by microscopic analysis of sample smears, which is a rapid method of detecting acid-fast bacilli. ${ }^{3}$ However, this method is limited in sensitivity and the ability to identify infecting species. The culture method can be used efficiently to diagnose TB, but it is time consuming as it requires a minimum of four weeks. Therefore, a test that combines the rapidity of microscopy and sensitivity of bacterial culture methods would facilitate easy diagnosis at slaughter houses and establishments requiring definite results at the shortest possible time. The test can be a valuable tool in veterinary diagnostics. In our study, there was efficient amplification of the LAMP system to detect MTC from suspected specimens from cattle and lechwe. These results clearly demonstrate the high sensitivity of the LAMP system in detecting TB as observed by others who have been working on human specimens.11,17,21 This result also suggests that LAMP may be superior to all procedures being used at the moment to detect TB in animals. One sample was detected as positive on LAMP although negative on culture. This could be attributed to low numbers of MTC viable cells in the section of the specimen under investigation. M. bovis was identified from the results of the multiplex-PCR, which targets three genetic regions that are $c f p 32$, RD9 and RD12.6,22-24

In summary, our study clearly provides evidence that the LAMP assay is a method that allows direct detection of MTC from processed clinical/abattoir specimens. This means that the technique can also be applied in the field of veterinary medicine for rapid confirmation of suspected cases. The ease of use and high sensitivity of this assay may facilitate the diagnosis and confirmation of MTC at slaughter houses, hence improving surveillance and control of TB at herd levels and in veterinary medicine in general.

\section{References}

1. de la Rua-Domenech R, Goodchild AT, Vordermeier HM, et al. Ante mortem diagnosis of tuberculosis in cattle: a review of the tuberculin tests, gamma-interferon assay and other ancillary diagnostic techniques. Res Vet Sci 2006;81:190-210.

2. Schiller I, Vordermeier HM, Waters WR, et al. Comparison of tuberculin activity using the interferon-gamma assay for the diagnosis of bovine tuberculosis. Vet Rec 2010:167:322-36.

3. Kent BD, Kubica GP. Public Health Mycobacteriology: a Guide for the Level III Laboratory. Atlanta: U.S. Department of Health and Human services, Centers for Disease Control, 1985.

4. Aranaz A, Cousins D, Mateos A, Dominquez, L. Elevation of Mycobacterium tuberculosis subsp. caprae Aranaz et al. 1999 to species rank as Mycobacterium caprae comb. Nov., sp. Nov. Int J Syst Evol Micr 2003;53:1785-9.

5. Cousins DV, Bastida R, Cataldi A, et al. Tuberculosis in seals caused by a novel member of the Mycobacterium tuberculosis complex: Mycobacterium pinnipedii sp. Nov. Int J Syst Evol Micr 2003;53:1305-14.

6. Huard RC, Fabre M, Haas PD, et al. Novel genetic polymorphisms that further delineate the phylogeny of the Mycobacterium tuberculosis complex. J Bacteriol 2006;188:4271-87.

7. Cosivi 0, Grange JM, Daborn CJ, et al. Zoonotic tuberculosis due to Mycobacterium bovis in developing countries. Emerg Infect Dis 1998;4:59-70.

8. Michel AL. Implications of tuberculosis in African wildlife and livestock. Ann NY Acad Sci 2002;969:251-5.

9. Pandey GS. Studies of the infectious diseases of the Kafue lechwe (Kobus lechwe kafuensis) with particular reference to tuberculosis in Zambia. PhD thesis, Azabu, University, Tokyo, Japan, 1998.

10. Notomi T, Okayama H, Masubuchi H, et al. Loop-mediated isothermal amplification of DNA. Nucleic Acids Res 2000;28:E63.

11. Iwamoto T, Sonobe T, Hayashi K. Loopmediated isothermal amplification for direct detection of Mycobacterium tuberculosis complex, M. avium and M. intracellulare. J Clin Microbiol 2003;41:2616-22.

12. Kuboki N, Inoue N, Sakurai T, et al. Loopmediated isothermal amplification for detection of African trypanosomes. J Clin Microbiol 2003;41:5517-24.

13. Parida M, Posadas G, Inoue GPS, Hasebe F, Morita K. Real-time reverse transcription loop-mediated isothermal amplification for rapid detection of West Nile virus. J Clin Microbiol 2004;42:257-63.

14. Parida M, Horioke $\mathrm{K}$, Ishida $\mathrm{H}$, et al. Rapid detection and differentiation of dengue virus serotypes by a real-time reverse transcription-loop mediated isothermal amplification assay. $\mathrm{J}$ Clin Microbiol 2005;43:2895-903.

15. Kimura H, Ihara M, Enomoto Y, et al. Rapid detection of herpes simplex virus DNA in cerebrospinal fluid: comparison between loop-mediated isothermal amplification and real-time PCR. Med Microbiol Immun 2005;194:181-5.

16. Yoda T, Suzuki Y, Yamazaki K, et al. Evaluation and application of reverse transcription loop-mediated isothermal amplification for detection of noroviruses. J Med Virol 2007;79:326-34.

17. Pandey BD, Poudel A, Yoda T, et al. Development of an in-house loop-mediated isothermal amplification (LAMP) assay for detection of Mycobacterium tuberculosis and evaluation in sputum samples of Nepalese patients. J Med Microbiol 2008;57:439-43.

18. Gracey JF, Collins DS, Huey J. eds. Meat Hygiene. 10th edn. Toronto: WB Saunders \& Company, 1999.

19. Suzuki Y, Katsukawa C, Inoue K, et al. Mutations in rpoB gene of rifampicin resistant clinical isolates of Mycobacterium tuberculosis in Japan. Kansenshogaku Zasshi 1995;69:413-19.

20. Nakajima C, Rahim Z, Fukushima Y, et al. Identification of Mycobacterium tuberculosis clinical isolates in Bangladesh by a species distinguishable multiplex PCR. BMC Infect Dis 2010;10:118.

21. Boehme CC, Nabeta P, Henostroza G, et al. Operational feasibility of using loop mediated isothermal amplification for diagnosis of pulmonary tuberculosis in microscopy centers of developing countries. J Clin Microbiol 2007;45:1936-40.

22. Brosch R, Gordon SV, Marmiesse M, et al. A new evolutionary scenario for the Mycobacterium tuberculosis complex. Proc Natl Acad Sci USA 2002;99:3684-9.

23. Huard RC, Lazzarini LC, Butler WR, Van Soolingen D, Ho JL. PCR based method to differentiate the subspecies of the Mycobacterium tuberculosis Complex on the basis of genomic deletions. J Clin Microbiol 2003;41:1637-50.

24. Huard RC, Chitale S, Leung M, et al. The Mycobacterium tuberculosis complex restricted gene cfp32 encodes an expressed protein that is detectable in tuberculosis patients and is positively correlated with pulmonary interleukin-10. Infect Immun 2003;71:6871-83. 